Method for making a single-step etch mask for 3D monolithic nanostructures

This content has been downloaded from IOPscience. Please scroll down to see the full text. 2015 Nanotechnology 26505302

(http://iopscience.iop.org/0957-4484/26/50/505302)

View the table of contents for this issue, or go to the journal homepage for more

Download details:

IP Address: 130.89.45.231

This content was downloaded on 23/02/2016 at $14: 43$

Please note that terms and conditions apply. 


\title{
Method for making a single-step etch mask for 3D monolithic nanostructures
}

\author{
D A Grishina ${ }^{1}$, C A M Harteveld ${ }^{1}$, L A Woldering ${ }^{2}$ and W L Vos ${ }^{1}$ \\ ${ }^{1}$ Complex Photonic Systems (COPS), MESA+ Institute for Nanotechnology, University of Twente, \\ PO Box 217, 7500 AE Enschede, The Netherlands \\ ${ }^{2}$ Transducers Science and Technology (TST), MESA+ Institute for Nanotechnology, University of \\ Twente, P.O. Box 217, 7500 AE Enschede, The Netherlands
}

E-mail: d.grishina@utwente.nl

Received 17 June 2015, revised 11 August 2015

Accepted for publication 29 September 2015

Published 17 November 2015

\begin{abstract}
Current nanostructure fabrication by etching is usually limited to planar structures as they are defined by a planar mask. The realization of three-dimensional (3D) nanostructures by etching requires technologies beyond planar masks. We present a method for fabricating a 3D mask that allows one to etch three-dimensional monolithic nanostructures using only CMOS-compatible processes. The mask is written in a hard-mask layer that is deposited on two adjacent inclined surfaces of a Si wafer. By projecting in a single step two different 2D patterns within one 3D mask on the two inclined surfaces, the mutual alignment between the patterns is ensured. Thereby after the mask pattern is defined, the etching of deep pores in two oblique directions yields a three-dimensional structure in $\mathrm{Si}$. As a proof of concept we demonstrate 3D mask fabrication for three-dimensional diamond-like photonic band gap crystals in silicon. The fabricated crystals reveal a broad stop gap in optical reflectivity measurements. We propose how 3D nanostructures with five different Bravais lattices can be realized, namely cubic, tetragonal, orthorhombic, monoclinic and hexagonal, and demonstrate a mask for a 3D hexagonal crystal. We also demonstrate the mask for a diamond-structure crystal with a 3D array of cavities. In general, the 2D patterns on the different surfaces can be completely independently structured and still be in perfect mutual alignment. Indeed, we observe an alignment accuracy of better than $3.0 \mathrm{~nm}$ between the 2D mask patterns on the inclined surfaces, which permits one to etch welldefined monolithic 3D nanostructures.
\end{abstract}

Keywords: nanotechnology, three-dimensional nanostructures, nanofabrication, etch mask, photonic band gap crystals, silicon photonics, integrated circuits

(Some figures may appear in colour only in the online journal)

\section{Introduction}

Progress in nanofabrication techniques is a key factor that enables the rapid growth of nanotechnology and its applications, because nanostructured materials exhibit unique complex behaviour. While much effort has been devoted to developing one-dimensional (1D) and two-dimensional (2D)

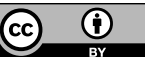

Content from this work may be used under the terms of the Creative Commons Attribution 3.0 licence. Any further distribution of this work must maintain attribution to the author(s) and the title of the work, journal citation and DOI. structures [1-3], opportunities for three-dimensional (3D) structures-such as a 3D photonic bandgap, sensing, optoelectronics and nano-electronics [4-6]-have been less explored because of the challenges in their realization. An important additional demand for 3D nanofabrication is to use only CMOS compatible techniques to allow for applications in micro and nano-electronics [7, 8]. Additional requirements to $3 \mathrm{D}$ nanostructures are high purity, and in the case of nanophotonic structures, a high refractive index contrast and a low roughness.

In this paper we concentrate on the fabrication of 3D nanophotonic structures and in particular 3D photonic 
crystals. 3D photonic crystals have already been realized using a large variety of techniques [9-14]. Firstly, one popular way to fabricate 3D nanostructures is the family of template-assisted methods [15-17]. In these methods, one first assembles a template that is infiltrated with a high refractive index material, followed by removal of the template by calcination or etching. A large variety of templates has been demonstrated, such as artificial opals made from colloidal nanoparticles (usually polymer or silica), polymer photoresist structured by 3D holography [18], or resist structured by direct laser writing (DLW) [19-21]. The geometry of the structures fabricated with DLW is well defined and can be very complex. Nevertheless, subsequent inversion to a high refractive index material introduces undesirable yet unavoidable impurities, roughness and undesired absorption [22]. Secondly, impressive structures have been created using layer-by-layer methods [23, 24]. Remarkable results have been reported on the fabrication of woodpile photonic crystal structures $[25,26]$. The main difficulty of layer-by-layer fabrication is the alignment between layers: each layer has to be carefully aligned with respect to the previous one. Here we call such an alignment a planar alignment since the layers that are being aligned are in parallel planes. In the CMOS industry the parameter characterizing the planar alignment between two layers is called overlay [27]. For the fabrication of an $N$ layer thick structure $N-1$ alignment steps are needed with $N-1$ overlay requirements. In order to avoid a large number of alignment steps a third class of fabrication methods has been proposed, where 3D nanostructures are created by consecutively patterning an etch mask on only two adjacent oblique wafer surfaces with only a single alignment step $(n=$ 1), followed by deep etching [28-30]. It is important to highlight that in this case the alignment is not planar anymore, as the masks that are aligned with respect to each other are lying in oblique planes. For the diamond-like photonic crystal under study, the out-of-plane alignment should be better than $50 \mathrm{~nm}$ [29]. In practice an out-of-plane alignment of $15 \mathrm{~nm}$ was reached [28]. Nevertheless, even one such out-of-plane alignment step introduces a significant complexity in the fabrication procedure, introducing deviations from perfect alignment and extending the time needed for fabrication.

In this paper we present a new fabrication method for monolithic 3D nanostructures. The key step is to define and make a single-step etch mask on two inclined surfaces simultaneously with an out-of-plane alignment ensured at the design stage. As an example for a single-step etch mask, we realize a pattern that yields a (110) plane of a cubic inverse woodpile structure on one surface and a (110) plane on a perpendicular adjacent surface. We describe the fabrication process of such a so-called '3D mask', as well as the subsequent etching process to fabricate $3 \mathrm{D}$ photonic band gap crystals with a diamond-like inverse woodpile structure (see figure 1). We investigate the ensured alignment at the design stage by carefully characterizing the realized structures on the inclined surfaces. We study the nanophotonic behaviour of the fabricated crystal by reflectivity measurements. We discuss other $3 \mathrm{D}$ structures that are feasible using our method such as disordered structures and different 3D Bravais

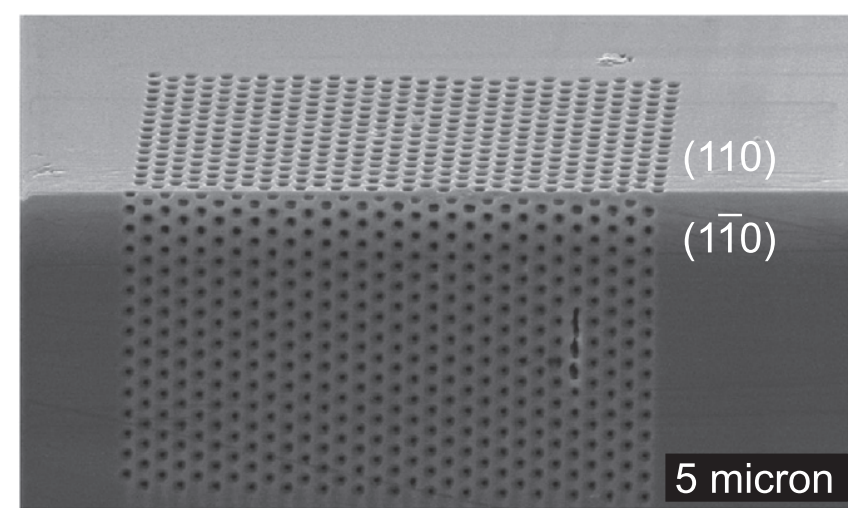

Figure 1. SEM image of a monolithic 3D photonic band gap crystal fabricated in Si using a single-step etch mask. The crystal has the inverse woodpile structure with a cubic diamond-like symmetry that consists of two sets of perpendicular pores. The top surface in the image is the (110) crystal plane and the perpendicular surface at the bottom is the (110) crystal plane. The scale bar is shown in the image.

lattices; as a proof of principle, we demonstrate a 3D mask for a hexagonal 3D nanostructure, and a 3D cavity array. Finally we discuss the use of various lithography techniques with our method.

\section{Fabrication process for the 3D single-step etch mask with built-in alignment}

The generic scheme to fabricate a 3D etch mask on two inclined surfaces is shown in figure 2. First, the hard mask material that serves as an etch stop is deposited on two inclined surfaces, see figure 2(a). Next, the desired pattern is projected onto the oblique surfaces, as shown in figure 2(b). The projection is made from a side such that both inclined surfaces can be reached. The projected pattern consists of two parts: pattern $a$, designed for one surface and pattern $b$, designed for the second surface. We emphasize that our method allows for a complete freedom to independently design the two patterns $a$ and $b$. In figure 2(b) the two patterns are designed to be similar. The two patterns are written in one projection and are therefore by design in perfect mutual alignment. Mask apertures for subsequent etching are opened during the third step of the mask fabrication process (figure 2(c)).

The fact that the pattern is projected on a non-normal surface must be taken into account in the pattern design. The pattern is designed in such a way that after projection on the inclined surfaces it yields the desired structure. As is shown in figure 2(b), the $x^{\prime}$ and $y^{\prime}$ coordinates in the design plane differ from the $x$ and $y$ coordinates on the sample surface.

We demonstrate our fabrication process using as an example a 3D cubic diamond-like photonic band gap crystal made from silicon. Due to its physical properties silicon plays an important role both in optics and electronics. As a material that is widely used in research and manufacturing, silicon is widely available, cheap, and has a very high purity. Among 
a)

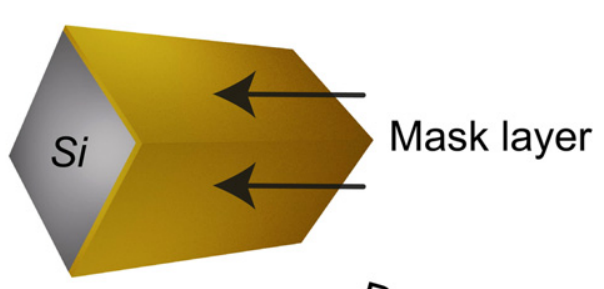

b)

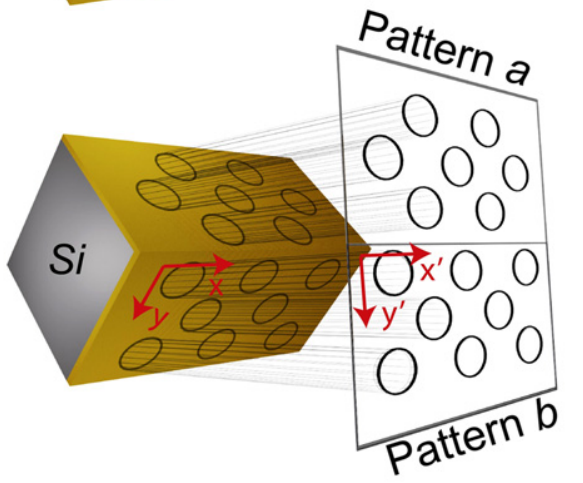

c)

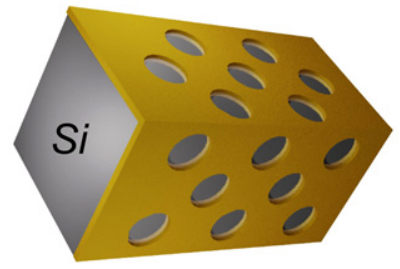

Figure 2. Scheme for the single-step etch mask fabrication on two perpendicular surfaces. (a) Deposition of a hard mask layer on two inclined surfaces of a Si wafer. (b) Patterning of a mask layer in one step on both surfaces-projection of a single 2D mask on a 3D surface. Patterns for both perpendicular surfaces are written in one projection and therefore alignment is ensured. (c) Apertures are opened in the mask layer to obtain an etch mask for two intersecting 2D structures that yield the desired 3D structure.

the diamond-like photonic band gap structures [31] we chose the inverse-woodpile photonic crystal. Inverse-woodpile photonic crystals deserve particular attention in view of the broad band gap with relative width $\left(\Delta \omega_{\text {gap }} / \omega_{\text {gap }}\right)$ of more than $25 \%$ [29, 32, 33]. These crystals consist of two mutually perpendicular rectangular arrays of cylindrical pores etched in a high refractive index material. Conceptually the fabrication of an inverse-woodpile structure is easy, although its fabrication remains a challenge due to the required precise alignment of the perpendicular sets of pores [28, 29, 34].

We start the fabrication procedure from a single crystalline Si wafer. We fabricated the 3D etch mask on two polished adjacent perpendicular surfaces of the wafer. In the first step we deposited a $50 \mathrm{~nm}$ thick Cr layer that serves as a hard mask material on two adjacent surfaces of a wafer (figure 2(a)). We choose $\mathrm{Cr}$ as a hard mask material due to its sustainability to $\mathrm{SH}_{6}$ etching [35], but other possible mask materials such as $\mathrm{SiN}$ or $\mathrm{SiC}$ are also compatible with our method. The deposition of $\mathrm{Cr}$ is done in a home-built sputtering machine and takes around $4 \mathrm{~min}$ for a $50 \mathrm{~nm}$ thick layer.

The patterning of the etch mask can be performed using several types of lithography [36] such as e-beam, focused ion beam (FIB), deep UV (DUV) step-and-scan or nanoimprint.

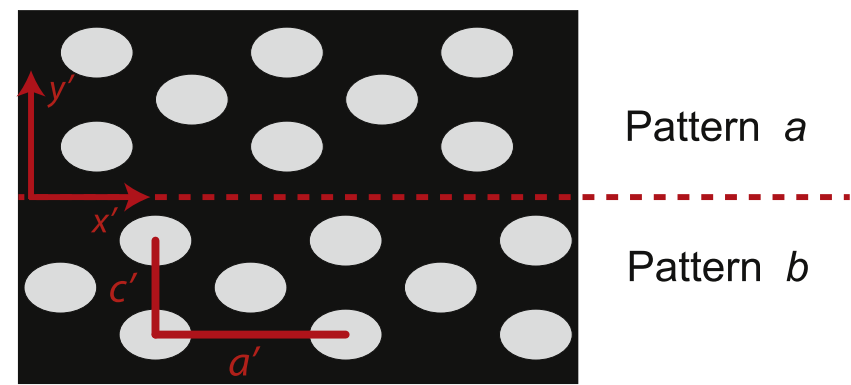

Figure 3. Geometry of the pattern that is projected on two inclined surfaces. The structure is designed to consist of two centred rectangular arrays shifted with respect to each other by $x^{\prime}=a^{\prime} / 4$ such that after being projected on two $45^{\circ}$ inclined surfaces it gives the (110) and (110) faces of a cubic inverse woodpile photonic crystal with lattice parameters $a=\sin 45^{\circ} \times a^{\prime}$ and $c=c^{\prime}$. The basic building blocks are designed to be elliptical, in order to yield circles on the mask after projection. The dashed line delimits the top part of the pattern (pattern $a$ ) that is projected on one surface of the wafer and the bottom part (pattern $b$ ) that is projected on the second surface.

Since the patterning of the etch mask is performed under an angle $\theta$, the depth of focus $F$ of a lithography tool will limit the mask size along the $y$-direction (figure 2(b)) to $\Delta y=F / \sin \theta$. For e-beam lithography the depth of focus can be as large as $1.5 \mu \mathrm{m}$ with a $100 \mathrm{~nm}$ pitch, depending on the magnification, working distance and aperture size [37, 38]. For DUV lithography the depth of focus depends notably on the pitch size and the wavelength of the UV source. For photonic applications considered here the length scales are in the order of the wavelength of light which is much larger (about $20 \times$ ) than the typical length scale in electronic integrated circuits and therefore a micron-sized depth of focus may be expected. In the case of nanoimprint lithography (NIL) the stamp can be fabricated using e-beam lithography and later transferred into a conformal mask pattern as described for example in [39]. In our case we had focused ion beam (FIB) milling equipment at our disposition to project the mask and open the apertures. FIB milling is known for its large depth of focus that exceeds $F=10 \mu \mathrm{m}$ [37]. We placed a sample in a FEI Nova 600 Nanolab FIB chamber under $45^{\circ}$ angle with respect to the ion beam gun so that both adjacent surfaces of the wafer can be reached (figure 2(b)).

The design of a single pattern that is projected on two adjacent surfaces consists of two parts as shown in figure 3 : pattern $a$ intended for one surface and pattern $b$ intended for the second surface. In our case the surfaces are orthogonal to each other and aligned at $45^{\circ}$ angle with respect to the ion beam gun in the $y$-direction (figure 2(b)). In the pattern design it is taken into account that projection of the pattern is made under an angle $\theta=45^{\circ}$ to the surfaces, meaning that $x^{\prime}$ and $y^{\prime}$ coordinates in the pattern design are related to the $x$ and $y$ coordinates on a surface of fabricated mask as the following: $x^{\prime}=x$ and $y^{\prime}=y / \sin \theta$. In order to form a cubic diamondlike structure inside the silicon wafer we patterned each surface of the wafer with a centred rectangular array of holes with lattice parameters $a$ and $c$, where $\frac{a}{c}=\sqrt{2}$ to fulfil the criterion for a cubic crystal. Arrays of holes for two surfaces 
are shifted by $c / 4$ in the $x$-direction. The pattern is shown in figure 3 and yields (110) and (110) crystal surfaces on a surface of a wafer. The mask pattern is projected on both surfaces in one step. Since the two patterns intended for different surfaces are contained in one image, the alignment between them is ensured. A patterning of a 3D mask consisting of two arrays of 30 by 30 holes each takes $7 \mathrm{~min}$.

After the etch mask is created, the next step is to etch deep pores inside Si through the openings. Etching can be done using a variety of techniques [40] such as reactive ion etching (RIE) [41], cryogenic etching, wet etching, photoelectrochemical etching [42, 43] or other types of etching depending on a desired structure and mask material. Nanopores are first etched in one direction, then the sample is rotated by $90^{\circ}$ and pores are etched in the second perpendicular direction. We etched deep nanopores using a deep reactive ion etcher (DRIE) Adixen AMS 100SE as described in [41]. It has been shown earlier that the air-silicon interface deflects ions by only a small angle [34]. Moreover for diamond-like inverse woodpile 3D photonic crystal it has been shown that its band gap is robust to deviations of the pore directions [44]: even with misalignments as large as $5^{\circ}$, the relative band gap width is only reduced from $24 \%$ to $21 \%$. Since we etch both sets of pores one after another we introduce an additional oxygen plasma cleaning step after etching each set of pores. The cleaning step is needed to remove the protective polymer layer remaining after the etching process into the front surface of the sample.

\section{Results and discussion}

\subsection{Fabricated structure characterization}

The result of patterning a 3D etch mask in a single step is shown in figure 4. Figure 4(a) shows four 3D masks in a row on the edge of a Si wafer. Each of the 3D masks was written in one step and consists of (110) and (110) crystal planes of the cubic inverse woodpile crystal. The number of 3D mask structures written along the edge of a Si wafer is only limited by the size of the wafer. The maximum width in the $x$ direction of a single structure that is written in one step is determined by the horizontal field of view of the lithography tool which is in our case a FIB setup. In this study the horizontal field of view was $12.8 \mu \mathrm{m}$ as set by the magnification of $10000 \times$. By decreasing the magnification or stitching the fields of view, it is possible to increase the size of a continuous structure in the $x$-direction. Thus, we may effectively consider the four closely spaced 3D patterns in figure 4(a) as one large $L_{x}=40 \mu \mathrm{m}$ sized nanostructure. The size in the $y$ direction is limited by the depth of focus of the tool and can be extended by performing for example multiple milling runs at different depths.

Figure 4(b) shows one complete mask patterned on both wafer surfaces. Above the dashed red line there is a surface that contains pattern $a$ corresponding to a (110) crystal plane of the targeted photonic crystal. Below the dashed line there is a perpendicular surface that contains pattern $b$ corresponding a)

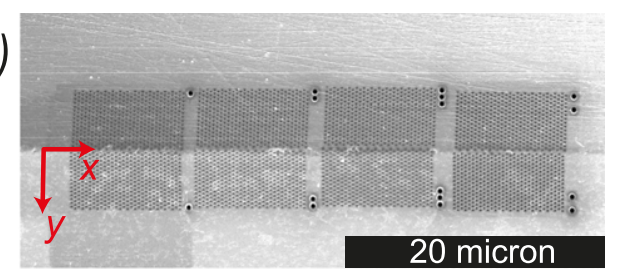

b)
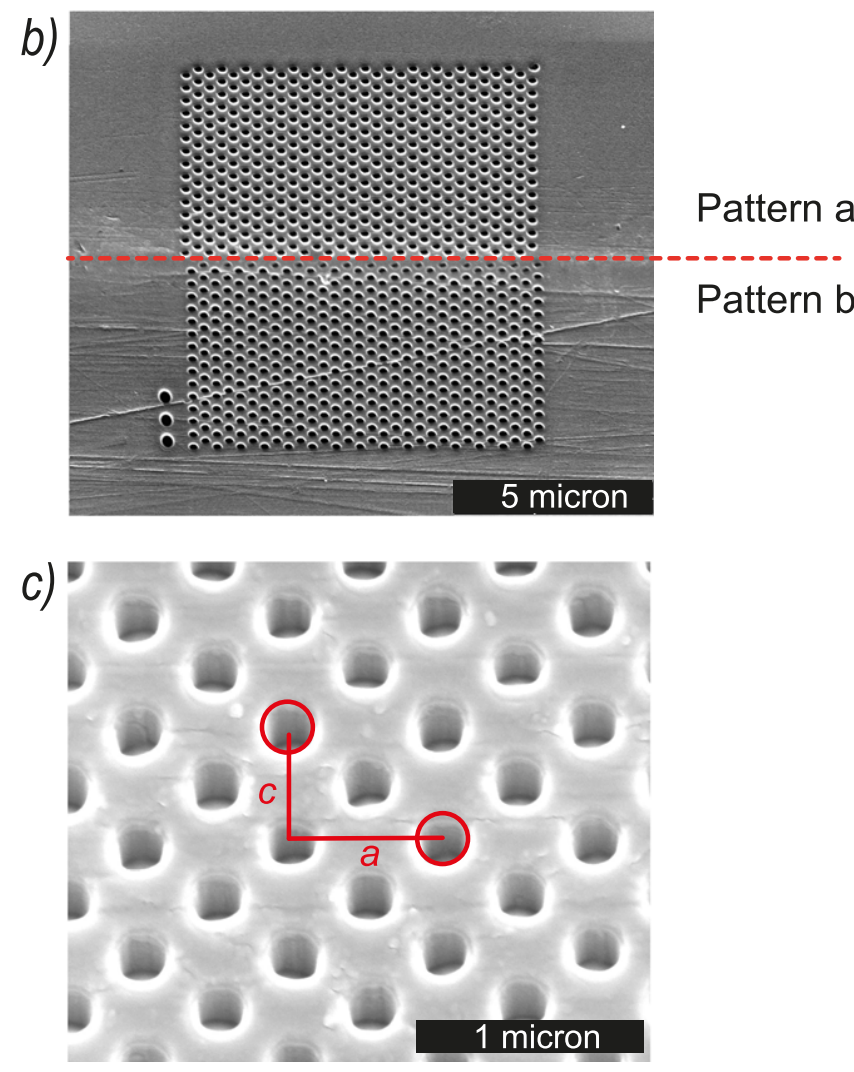

Figure 4. (a) Overview of a Si wafer with four $3 \mathrm{D}$ etch masks milled in one step. The coordinates $(x, y)$ are indicated. (b) Side view on one of the mask patterns. The dashed line in the middle indicates the 90 degree edge of the Si wafer. (c) Zoom-in on one surface of a mask pattern. $a$ and $c$ are lattice parameters with $\frac{a}{c}=\sqrt{2}$. Scale bars are shown in each image.

to a $(1 \overline{1} 0)$ crystal surface. The design for this mask shown in figure 3 is such that centres of the apertures in pattern $b$ are shifted by $\Delta x=a / 4$ to be exactly in the middle between apertures of pattern $a$. The lower surface of the wafer has some deep lines that are the result of manual polishing and which slightly reduce the quality of the mask layer on that side. Fortunately, the effect of such lines was found to be insignificant in subsequent processing, although it may introduce optical scattering.

Since patterns $a$ and $b$ are located on oblique surfaces we characterize the alignment between patterns $a$ and $b$ that we refer to as the out-of-plane alignment. We foresee two sources of possible misalignment: first, the sample may be placed under an angle different from $45^{\circ}$ during the mask projection step (figure 2(b)); second, the mask pattern can be rotated with respect to the edge of the sample. In the first case the sample tilt is precisely set by a positioning stage and 
a)

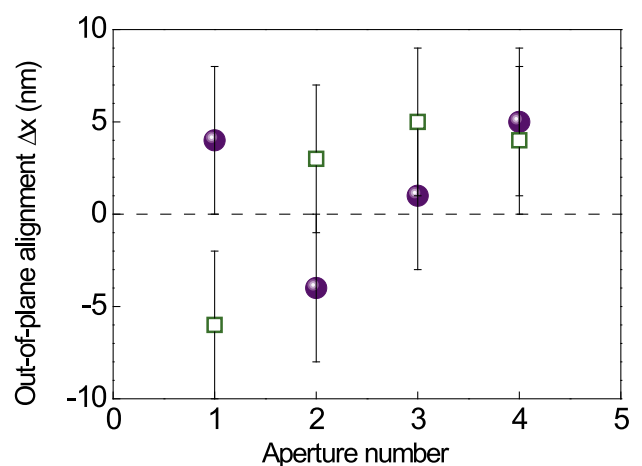

b)

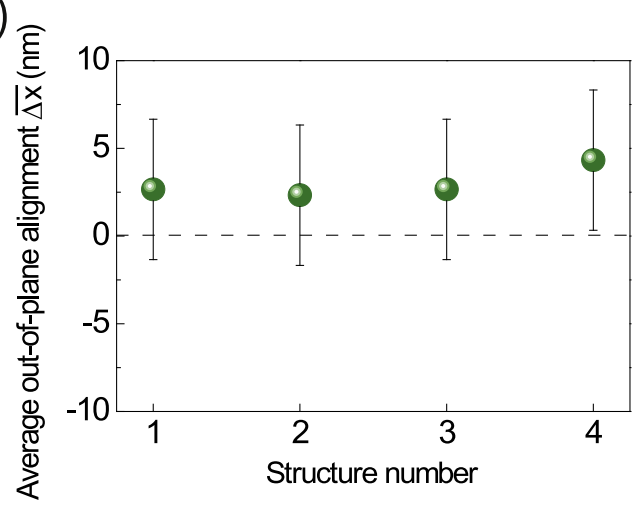

Figure 5. The out-of-plane alignment defined as a deviation $\Delta x$ of the position of an aperture in pattern $a$ relative to an aperture on the oblique surface in pattern $b$. The data were taken on the pattern shown in figure 9. (a) Circles show measurements taken for four pairs of apertures close to the wafer edge; apertures in pattern $a$ are located at $y=1.42 \mu \mathrm{m}$ and in pattern $b$ at $y=-1 \mu \mathrm{m}$. Squares show measurements for four pairs of apertures further from the edge; in pattern $a$ at $y=1.42 \mu \mathrm{m}$ and in pattern $b$ at $y=-5.42 \mu \mathrm{m}$. (b) Out-of-plane alignment averaged over four pairs of apertures $\overline{\Delta x}$ within one structure is shown for four different structures. In both figures error bars represent the resolution of the SEM.

controlled with an SEM image, therefore we expect the tilt alignment to be always better than a few degrees. For a misalignment by $2^{\circ}$ the dimensions of a projected pattern result in a structural strain of only $3 \%$. In the second case if the projected pattern is rotated with respect to the wafer edge by an angle $\delta$, the apertures located at a distance $y$ from the edge will be displaced by $\Delta x=y \cdot \sin \delta$ from their designed positions. In practice we expect the angular misalignment to be less than $\delta=2^{\circ}$, which is sufficiently small to realize bona fide $3 \mathrm{D}$ nanostructures.

To characterize the out-of-plane alignment we digitally take the grey value cross-sections from the SEM images through a row of apertures. First we select an aperture in pattern $a$ and take a grey value cross-section from the SEM image to define its central position. Then we select an aperture in pattern $b$, take a grey value cross-section to define its position and compare the relative position $\Delta x$ of these two apertures. In this way we determine a mutual alignment of the apertures located on inclined planes. In figure 5(a) we have collected two sets of data for the structure shown in figure 9. In the first set indicated as circles we plot the out-of-plane alignment between pairs of apertures where one aperture in pattern $a$ is taken in the row at a distance $y=1.4 \mu \mathrm{m}$ from the edge and the second aperture in pattern $b$ is taken in the row at a distance $y=-1 \mu \mathrm{m}$ from the edge. From the data we conclude that the deviation of apertures positions from the design is within the error bar of SEM accuracy. To verify the alignment over further distances from the edge, in the second set of alignment data, shown as squares in figure 5(a), we take the second aperture in pattern $b$ in a row located at $y=-5.42$ $\mu \mathrm{m}$ and compare their positions to the same apertures in pattern $a$ as before. We see that the out-of-plane alignment stays within the resolution of SEM for both sets of data, independent of the distance from the edge, thus providing an upper boundary of $0.05^{\circ}$ for rotational misalignment discussed earlier. We find here the deviation from the designed structure (figure 3 ) to be at most $5 \mathrm{~nm}$. In figure 5(b) we plot the out-of-plane alignment data $\overline{\Delta x}$ that are averaged over four pairs of apertures within one structure for four different structures (as illustrated e.g. in figure 4(a)). It is seen that the average over a mask varies between $\overline{\Delta x}=2.3 \mathrm{~nm}$ and $\overline{\Delta x}=4.3 \mathrm{~nm}$ with a mean of $3.0 \mathrm{~nm}$. The error bars in both figures 5(a) and (b) indicate the typical accuracy of $\pm 4 \mathrm{~nm}$ of the scanning electron microscope. Thus, we conclude that the out-of-plane alignment data are mostly determined by the SEM error and are better than $3.0 \mathrm{~nm}$. Therefore, the out-ofplane alignment for individual apertures is consistent with zero deviation, in agreement with the starting point of our 3D mask method that the two oblique patterns have built-in mutual alignment.

Figure 4(c) shows a zoom-in to the front surface of a wafer. We see that the elliptical apertures in the pattern design (see figure 3 ) have been correctly projected to become circular apertures with the diameter of $273 \mathrm{~nm}$ on the sample surface. From figures 4 and 5 we conclude that we have successfully fabricated a desired 3D mask structure on two inclined surfaces. We emphasize that the alignment between the patterns on two inclined planes is ensured at the design stage since both patterns are written in one projection and is within the resolution of SEM.

After etching of deep pores in silicon in two perpendicular directions, we have sacrificed one crystal in order to view the internal structure of the sample by milling it with a focused ion beam. Figure 6(b) shows a schematic representation of the cut and a surface plane that is open for viewing. The cut was made under a $45^{\circ}$ angle to both surfaces. Since the pore depth is finite, starting at a certain depth in the structure the perpendicular pores will not overlap inside the crystal. Pores that are far from the edge of a wafer are not deep enough in order to reach the corresponding perpendicular pore, see the green line in figure 6(c). Therefore we expect to see a region inside the crystal closer to the edge where pores overlap and form a 3D structure. Further from the edge we expect a region where the structure will be twodimensional. The SEM image of a crystal cross-section is shown in figure 6(a). We see that using a single-step etch mask deep pores were successfully etched in silicon. Pores etched in both perpendicular directions form a 3D diamondlike structure in the bulk. Far from the surface of the Si wafer the pores are not overlapping. In the present case, the depth of 
a)

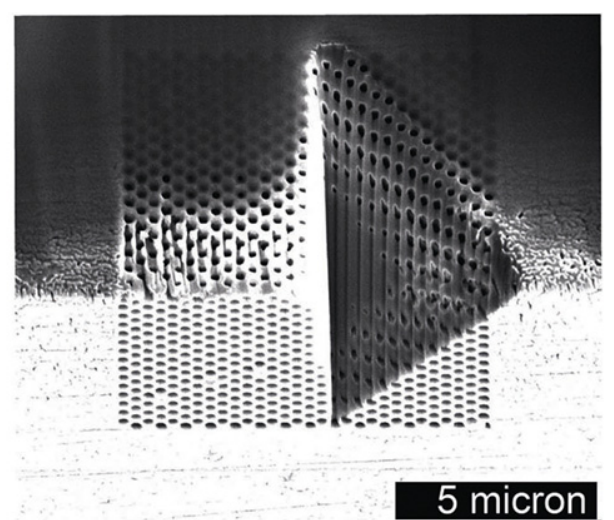

b)

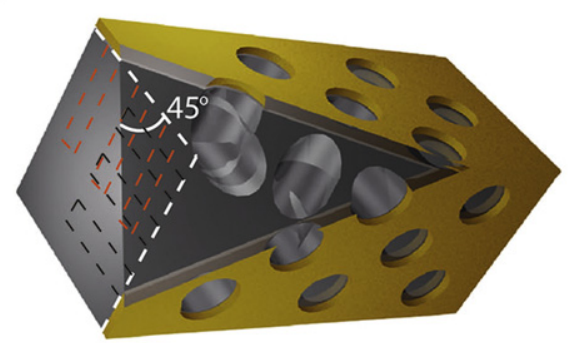

c)

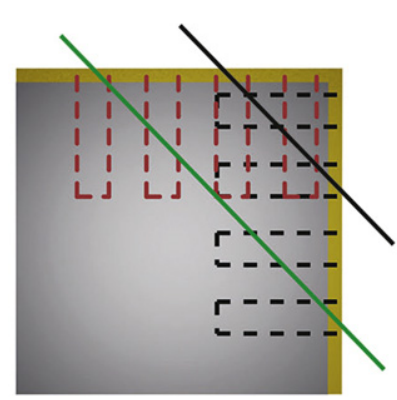

Figure 6. (a) The fabricated 3D diamond-like photonic crystal that was opened up by focused ion beam milling. Scale bar is shown in the picture. (b) Schematic representation of how a cross-section is milled on a fabricated sample. The cut is made at $45^{\circ}$ to the side of silicon bar. Dashed lines indicate the pore geometry. (c) Schematic cross-section that illustrates how limited pore depth appears on the milled structure. The black line shows where pores overlap with each other and the green line shows the depth beyond which the arrays of pores are two-dimensional.

the pores is determined to be $4 \mu \mathrm{m}$ deep with an aspect ratio (depth to width ratio) of 14 . The size of the fabricated photonic crystal is limited by the depth of the pores in the silicon [41]. We conclude from the cross-section that a single-step etch mask with ensured alignment allows us to fabricate a 3D monolithic nanostructure in bulk silicon.

\subsection{Nanophotonic behaviour}

To verify the nanophotonic behaviour of the fabricated inverse-woodpile photonic band gap crystal, we have performed reflectivity measurements perpendicular to the (110) surface of a crystal. We used a method described in [45]; in brief, a Fianium supercontinuum source was used as a broad band light source. The laser beam was focused on a sample

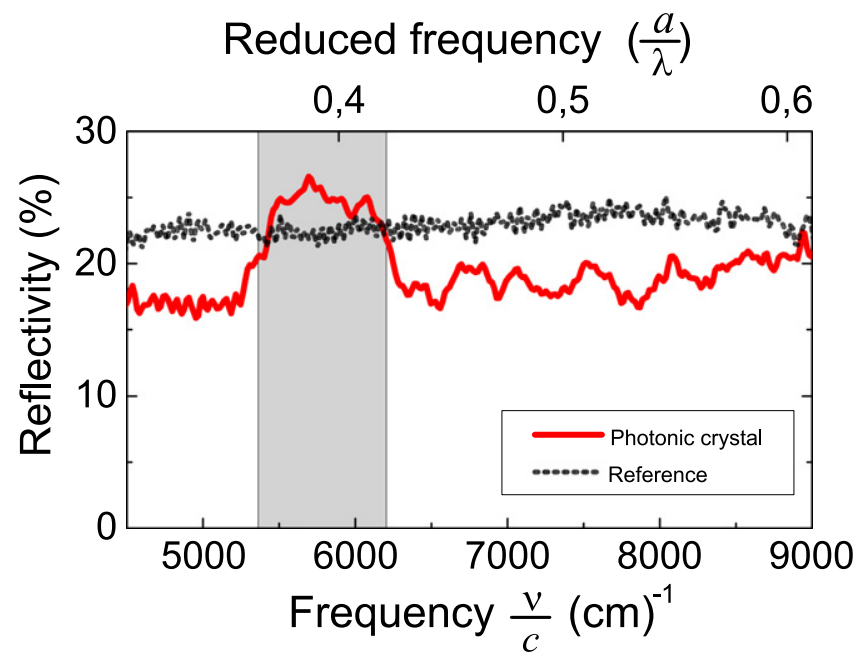

Figure 7. Reflectivity measurements on a fabricated 3D photonic crystal and a non-photonic reference. The grey bar indicates the stop gap calculated for a fabricated sample with lattice parameters taken from SEM images: $\frac{r}{a}=0.16$ and $a=679 \mathrm{~nm}$.

surface with a reflective objective with a numerical aperture $\mathrm{NA}=0.65$. Spectral measurements were performed using a Biorad 600 Fourier-transform infra-red (FTIR) spectrometer with a resolution of $16 \mathrm{~cm}^{-1}$. Figure 7 shows the measured reflectivity calibrated to a gold mirror. The experimental spectrum reveals a broad reflectivity peak from $5246 \mathrm{~cm}^{-1}$ to $6341 \mathrm{~cm}^{-1}$. The maximum reflectivity of $26 \%$ is currently limited by the finite size of a crystal, surface roughness and a relatively large beam size in the optical setup. The beam size during the experiment was close to $6 \mu \mathrm{m}$ which is larger than the crystal size. Taking into account that crystals are necessarily located at the edge of the silicon wafer, in the case when the beam size is larger than the crystal size, a significant amount of laser light shines into the air resulting in a low reflectivity value. The collected reflectivity spectrum averages over the illuminated area, therefore in the case when the beam size is larger that a crystal size, reflection from a bulk silicon gives rise to the reflectivity value outside the stop gap and therefore decreases its maximum value. Nevertheless, the reflectivity peak indicates nanophotonic behaviour of a photonic crystal fabricated using a single-step etch mask. The expected stop gap in the $\Gamma-Z$ direction according to plane wave band structure calculations in [45] is situated from $5362 \mathrm{~cm}^{-1}$ to $6204 \mathrm{~cm}^{-1}$. Parameters for the stop gap calculations were taken from SEM images of the fabricated sample $\left(\frac{r}{a}=0.16\right.$ and $\left.a=679 \mathrm{~nm}\right)$. Therefore, the observed stop band is in good agreement with the calculated stop gap for an infinite 3D photonic crystal. From observed nanophotonic behaviour of our sample we conclude that the functional photonic structure has been successfully fabricated using a 3D single-step etch mask.

\subsection{Structures feasible for fabrication}

There is a large variety of structures that can be fabricated using the presented technique. In general, mask patterns 

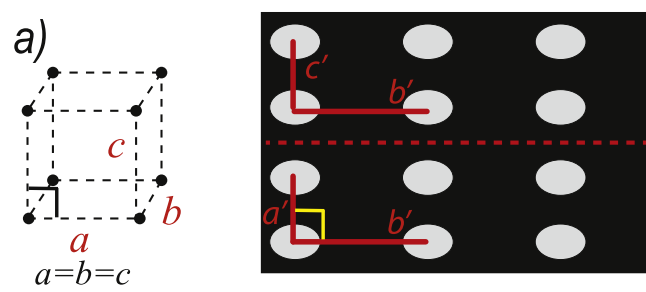

Pattern a

b)

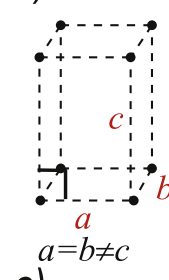

c)

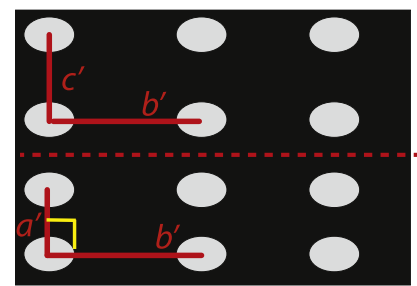

Pattern a

Pattern $b$
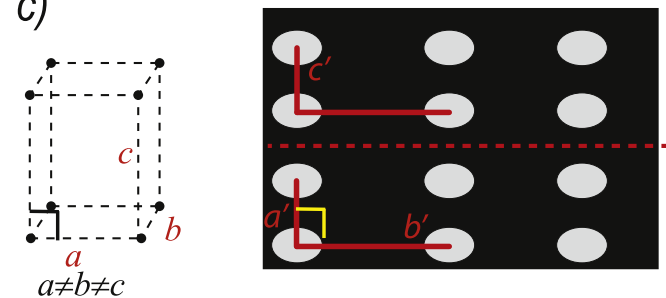

Pattern a

d)
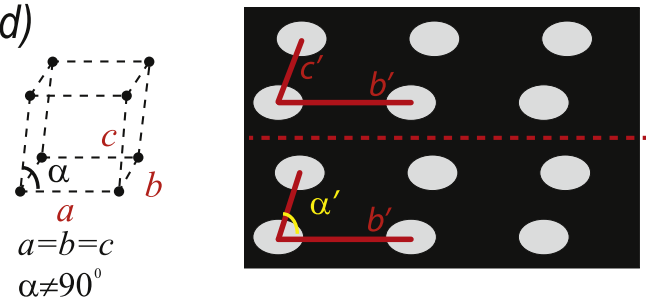

Pattern a

e)
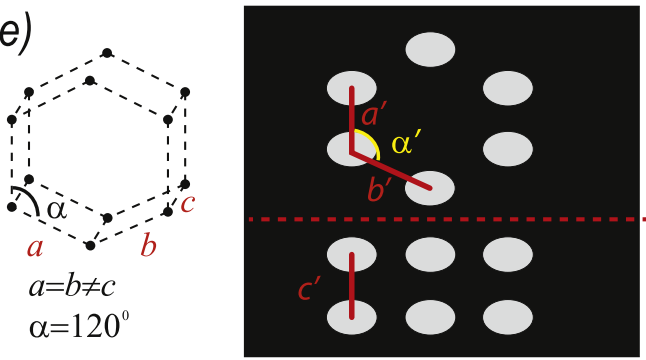

Pattern a

- - - - - - -

Pattern $b$

Figure 8. Pattern designs for different lattice system fabrication. The $3 \mathrm{D}$ scheme of the structure is shown on the left and corresponding pattern for the etch mask is on the right. (a) Cubic, (b) tetragonal, (c) orthorhombic, (d) monoclinic and (e) hexagonal.

projected in one step on inclined surfaces have an arbitrary structure and are still in perfect alignment with respect to each other. We consider 3D Bravais lattices that can be created with the described fabrication process. Using different pattern designs, it is possible to achieve structures with five Bravais lattice systems: cubic, monoclinic, orthorhombic, tetragonal and hexagonal. On the left side of figure 8(a) the simple cubic lattice is shown. On the right side there is a pattern design for the fabrication of a simple cubic structure, where all lattice parameters are equal $(a=b=c)$. Taking into account that the pattern is projected on $\theta=45^{\circ}$ inclined surfaces, in the design $a^{\prime}=a / \sin 45^{\circ}, c^{\prime}=c / \sin 45^{\circ}$ and $b^{\prime}=b$. This design consists of two rectangular arrays of apertures aligned with no

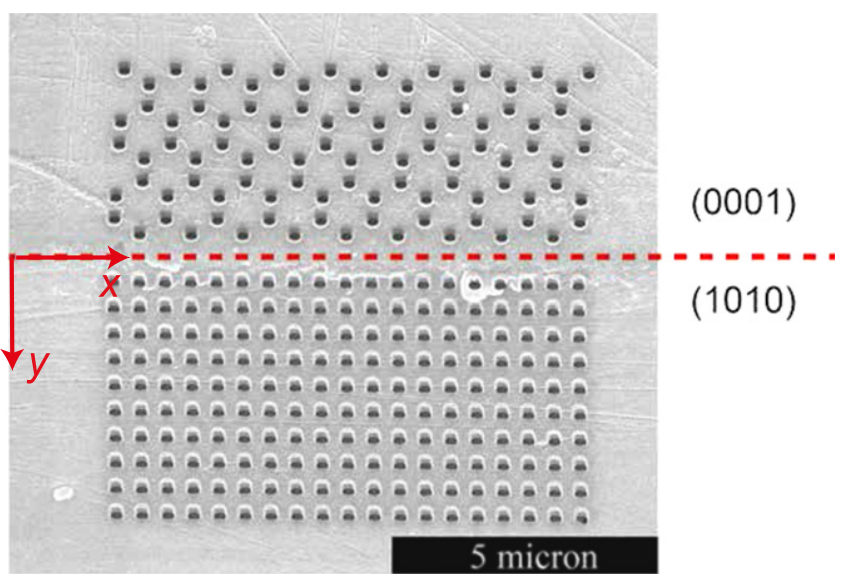

Figure 9. SEM image of a mask for hexagonal 3D crystal fabrication. The dashed line shows the $90^{\circ}$ edge of a wafer. The top side is a (0001) crystal plane of hexagonal structure and the bottom side is a (1010) crystal plane. The scale bar is shown on the picture.

shift with respect to each other. For completeness we note that this design for a cubic structure is not unique, as many different designs are feasible. In particular, the mask design presented in figure 3 confirms that there are multiple possible designs for cubic structure, notably depending on the type of cubic symmetry (simple versus face centred). Figure 8(b) shows a simple tetragonal structure on the left side and the design pattern for realization of such a structure on two perpendicular surfaces on the right side. In case of tetragonal structure $a=b \neq c$, therefore in the design pattern $c^{\prime} \neq a^{\prime}$. That means that the complete mask pattern for a tetragonal structure consists of two different rectangular arrays of apertures, one with lattice parameters $a^{\prime}$ and $b^{\prime}$ and other one with parameters $c^{\prime}$ and $b^{\prime}$. Figure 8(c) shows the orthorhombic structure on the left and the corresponding design pattern on the right. For an orthorhombic structure $a \neq b \neq c$, which means that in the design pattern all lattice parameters are different. Hence the complete design consists of two different rectangular arrays of apertures with parameters $a^{\prime}, b^{\prime}$ and $c^{\prime}$, $b^{\prime}$. On the left side of figure 8(d) the monoclinic structure is shown, where $a=b=c$ and the angle between two lattice vectors is $\alpha \neq 90^{\circ}$. In the pattern design this means that the rows of apertures are shifted with respect to each other so that the angle between vectors $a^{\prime}, b^{\prime}$ and $c^{\prime}, b^{\prime}$ is $\alpha^{\prime}=\alpha / \sin 45^{\circ}$. In figure $8(\mathrm{e})$ the hexagonal structure is shown on the left side and the pattern design on the right side. The structure consists of two hexagons shifted with respect to each other. Lattice parameters are $a=b \neq c$ and lattice angle $\alpha=120^{\circ}$. The pattern design consists of two completely different patterns. Pattern $a$ is similar to the well known hexagonal graphenelike pattern [46], whereas pattern $b$ has a rectangular array of apertures. Due to projection on the inclined surfaces the lattice parameters in the design are $a^{\prime}=a / \sin 45^{\circ}$, $c^{\prime}=c / \sin 45^{\circ}$ and $b^{\prime}=b / \cos \alpha^{\prime}$, where $\alpha^{\prime}=\alpha / \sin 45^{\circ}$. The hexagonal mask has been realized in $\mathrm{Cr}$ on a $\mathrm{Si}$ wafer by means of a fabrication procedure described earlier in this paper and shown in figure 9. The red dashed line shows the $90^{\circ}$ edge of a wafer. The top side on the picture is the (0001) 


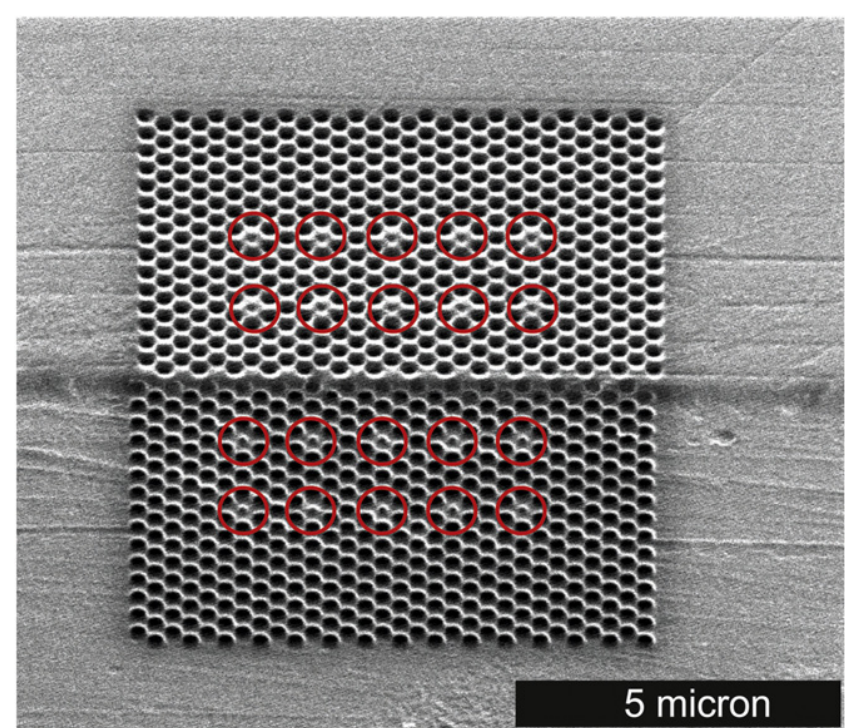

Figure 10. SEM image of a mask for an array of $2 \times 2 \times 4$ cavities in a $3 \mathrm{D}$ photonic band gap crystal. Smaller pores that form cavities inside the structure are marked with red circles.

crystal surface of hexagonal structure and the bottom side is a (1010) crystal plane. The 3D mask for this hexagonal structure shows clearly the flexibility of the fabrication method to realize structures with independent patterns on inclined surfaces with an unprecedented out-of-plane alignment better than $5 \mathrm{~nm}$. All described 3D structures, except cubic, are predicted to reveal sub-Bragg diffraction [47] which makes them an interesting subject for optical study, moreover, simple cubic and hexagonal structures have been predicted to reveal 3D band gaps [48, 49].

Besides different periodic structures it is possible to fabricate masks that yield non-periodic three-dimensional structures or periodic structures with controlled defects. An interesting example is the fabrication of a 3D photonic band gap crystal with a cavity inside. The geometry of the cavity described in [50] can be straightforwardly realized with the presented fabrication method by making two apertures for crossing pores on oblique planes smaller. Moreover, it is possible to realize an array of such cavities in a $3 \mathrm{D}$ photonic crystal. In figure 10 the mask pattern for an array of $2 \times 2 \times 4$ cavities in $3 \mathrm{D}$ photonic crystal is shown. The resulting $3 \mathrm{D}$ array of band-gap cavities would represent the photonic version of the Anderson tight-binding model [51] that may reveal intricate nanophotonic phase transitions for light. Another example is the fabrication of three-dimensional disordered or aperiodic structures. In this case patterns $a$ and $b$ can be arrays of randomly distributed apertures or incommensurable lattices [52].

Since patterns $a$ and $b$ are independent from each other, it is also possible to fabricate functionally different components on inclined surfaces. Figure 1 in [28] shows an illustration of a suggested chip consisting of two integrated circuits on adjacent surfaces that are interconnected. Using the presented fabrication method it is possible to make an interconnection between different integrated circuits with ensured alignment.
In addition, it is possible to project an optically functional device on one surface and electronic components on the other surface. Such architecture greatly increases the density of components on chip, makes interconnections between them easier and provides the possibility to spatially separate optics and electronics on chip.

The limitation for a number of possible structures comes from the subsequent silicon etching step. So far we assume that etching is always done with pores with direction normal to the surface of the sample. Nevertheless there are examples where etching under an angle has been demonstrated [53] which can further increase the number of feasible structures.

\section{Conclusions and outlook}

In summary, a novel method has been proposed to fabricate a three-dimensional etch mask in one step with built-in alignment. The out-of-plane alignment between structures onto oblique adjacent surfaces has been characterized by means of deviation from the designed structure. The out-of-plane alignment has been found to be better than $3.0 \mathrm{~nm}$. A threedimensional band gap photonic crystal with an inverse woodpile structure has been realized using a mask fabricated in a proposed way. The fabricated three-dimensional photonic crystal reveals a broad stop gap in optical reflectivity measurements. The mask designs for 3D nanostructures with five different Bravais lattices, namely cubic, tetragonal, orthorhombic, monoclinic and hexagonal have been shown. The mask for a 3D hexagonal structure and for a 3D array of cavities had been realized on a Si wafer.

The presented method allows fabrication of 3D nanostructures that enable exciting prospects in nanophotonics including the pursuit of cavity resonances in 3D photonic band gap crystals [50] and 3D Anderson localization of light in photonic crystals [51, 54]. The next step for further development of the single-step etch mask technique is to broaden it to different types of lithography discussed earlier. DUV lithography is particularly interesting since it is widely used in the CMOS industry and therefore opens new possibilities notably for 3D photonic integrated circuits $[55,56]$. Also, patterning of inclined surfaces with accurate alignment allows one to integrate on-chip different functional devices: for example, an optical device on one surface and an electronic device on the adjacent surface. We therefore foresee novel applications of our method in opto-electronics, integrated photonics and sensing.

\section{Acknowledgements}

The authors kindly thank Lyuba Amitonova for help in drawing the 3D pictures; Oluwafemi Ojambati, Maryna Meretska, as well as Arie den Boef, Jo Finders (ASML), Robert van de Laar, Jeroen Bolk, Huub Ambrosius, Meint Smit (TU Eindhoven) and William Green (IBM) for fruitful discussions; Andreas Schultz for help with chemical cleaning of samples; Jorge Perez-Vizcaino for help with reflectivity 
measurements; Willem Tjerkstra and Johanna van den Broek for early contributions; and Allard Mosk for encouragement. This work was supported by the 'Stirring of light!' programme of the Stiching voor Fundamenteel Onderzoek der Materie (FOM), which is financially supported by the Nederlandse Organisatie voor Wetenschappelijk Onderzoek (NWO) and by 'Stichting voor Technische Wetenschappen' (STW).

\section{References}

[1] Leuthold J, Koos C and Freude W 2010 Nonlinear silicon photonics Nat. Photonics 4 535-44

[2] Xia F, Wang H, Xiao D, Dubey M and Ramasubramaniam A 2014 Two-dimensional material nanophotonics Nat. Photonics 8 899-907

[3] Chrostowski L and Hochberg M 2015 Silicon Photonics Design: From Devices to Systems (Cambridge: Cambridge University Press)

[4] Santos A, Deen M J and Marsal L F 2015 Low-cost fabrication technologies for nanostructures: state-of-the-art and potential Nanotechnology 26042001

[5] Arpin K A, Mihi A, Johnson H T, Baca A J, Rogers J A, Lewis J A and Braun P V 2010 Multidimensional architectures for functional optical devices Adv. Mater $\mathbf{2 2}$ 1084-101

[6] Wehrspohn R B and Üpping J 2012 3D photonic crystals for photon management in solar cells J. Opt. 14024003

[7] Judy J W 2001 Microelectromechanical systems (MEMS): fabrication, design and applications Smart Mater. Struct. 10 $1115-34$

[8] Assefa S, Xia F, Green W M J, Schow C L, Rylyakov A V and $\mathrm{Yu}$ Vlasov 2010 CMOS-integrated optical receivers for onchip interconnects IEEE J. Sel. Top. Quantum Electron. 16 1376-85

[9] Vlasov Yu A, Xiang-Zheng B, James C S and Norris D J 2001 On-chip natural assembly of silicon photonic bandgap crystals Nature 414 289-93

[10] López C 2003 Materials Aspects of Photonic Crystals $A d v$. Mater. 15 1679-704

[11] Galisteo-López J F, Ibisate M, Sapienza R, Froufe-Pérez L S, Blanco Á and López C 2010 Self-assembled photonic structures Adv. Mater 23 30-69

[12] Braun P 2014 Materials chemistry in 3D templates for functional photonics Chem. Mater 26 277-86

[13] Ghulinyan M and Pavesi L (ed) 2015 Light Localisation and Lasing: Random and Quasi-Random Photonic Structures (Cambridge: Cambridge University Press)

[14] Vos W L and Woldering L A 2015 Cavity quantum electrodynamics with three-dimensional photonic band gap crystals Light Localisation and Lasing: Random and QuasiRandom Photonic Structures ed M Ghulinyan and L Pavesi (Cambridge: Cambridge University Press) ch 8

[15] Imhof A and Pine D J 1997 Ordered macroporous materials by emulsion templating Nature 389 948-51

[16] Holland B T, Blanford C F and Stein A 1998 Synthesis of macroporous minerals with highly ordered threedimensional arrays of spheroidal voids Science 281 538-40

[17] Wijnhoven J E G J and Vos W L 1998 Preparation of photonic crystals made of air spheres in titania Science 281 802-4

[18] Campbell M, Sharp D N, Harrison M T, Denning R G and Turberfield A J 2000 Fabrication of photonic crystals for the visible spectrum by holographic lithography Nature $\mathbf{4 0 4}$ 53-6
[19] Sun H B, Matsuo S and Misawa H 1999 Three-dimensional photonic structures achieved with two-photon-absorption photopolymerization of resin Appl. Phys. Lett. 74 786-8

[20] Cumpston B H et al 1999 Two-photon polymerization initiators for three-dimensional optical data storage and microfabrication Nature 398 51-4

[21] Deubel M, Wegener M, Kasao A and John S 2004 Direct laser writing and characterization of slanted pore photonic crystals Appl. Phys. Lett 85 1895-7

[22] Wijnhoven J E G J, Bechger L and Vos W L 2001 Fabrication and characterization of large macroporous photonic crystals in titania Chem. Mater. 13 4486-99

[23] Fleming J G and Lin S Y 1999 Three-dimensional photonic crystal with a stop band from 1.35 to $1.95 \mathrm{~m}$ Opt. Lett. 24 $49-51$

[24] Ogawa S, Imada M, Yoshimoto S, Okano M and Noda S 2004 Control of light emission by 3D photonic crystals Science 305 227-9

[25] Ogawa S, Ishizaki K, Furukawa T and Noda S 2008 Spontaneous emission control by 17 layers of threedimensional photonic crystals Electronics Lett. 44 377-8

[26] Noda S, Tomoda K, Yamamoto N and Chutinan A 2000 Full three-dimensional photonic bandgap crystals at near-infrared wavelengths Science 289 604-6

[27] Levinson H J 2001 Principles of Lithography (Bellingham, WA: SPIE) 363

[28] Tjerkstra R W, Woldering L A, van den Broek J M, Roozeboom F, Setija I D and Vos W L 2011 A method to pattern masks in two inclined planes for three-dimensional nano- and microfabrication J. Vac Sci. Technol. B 29 061604

[29] Woldering L A, Mosk A P, Tjerkstra R W and Vos W L 2009 The influence of fabrication deviations on the photonic band gap of three-dimensional inverse woodpile nanostructures J. Appl. Phys. 105093108

[30] Erdmanis M, Sievil P, Shah A, Chekurov N, Ovchinnikov V and Tittonen I 2014 Focused ion beam lithography for fabrication of suspended nanostructures on highly corrugated surfaces Nanotechnology 25335302

[31] Maldovan M and Thomas E L 2004 Diamond-structured photonic crystals Nature Mater. 3 593-600

[32] Ho K M, Chan C T, Soukoulis C M, Biswas R and Sigalas M 1994 Photonic band gaps in three dimensions: New layer-by-layer periodic structures Solid State Commun. 89 413-6

[33] Hillebrand R, Senz S, Hergert W and Göele 2003 Macroporous-silicon-based three-dimensional photonic crystal with a large complete band gap J. Appl. Phys. 942758

[34] van den Broek J M, Woldering L A, Tjerkstra R W, Segerink F B, Setija I D and Vos W L 2012 Inversewoodpile photonic band gap crystals with a cubic diamondlike structure made from single-crystalline silicon $A d v$. Funct. Mater. 22 25-31

[35] Williams K R, Gupta K and Wasilik M 2003 Etch rates for micromachining processing: II. J. Microelectromech. Syst. 12 761-78

[36] Imboden M and Bishop D 2014 Top-down nanomanufacturing Phys. Today 67 45-50

[37] Grivet P 1972 Electron Optics (Oxford: Pergamon) 505

[38] Dwir B, Utke I, Kaufman D and Kapon E 2000 Electron-beam lithography of V-groove quantum wire device Microelectron. Eng. 53 295-8

[39] Smith C L, Desiatov B, Giykmann I, Fernandez-Cuesta I, Levy U and Kristenses A 2012 Plasmonic V-groove waveguides with Bragg grating filters via nanoimprint lithography Opt. Express 20 5696-706

[40] Banqiu W, Kumar A and Pamarthy S 2010 High aspect ratio silicon etch: A review J. Appl. Phys. 108051101 
[41] Woldering L A, Tjerkstra R W, Jansen H V, Setija I D and Vos W L 2008 Periodic arrays of deep nanopores made in silicon with reactive ion etching and deep UV lithography Nanotechnology 19145304 1-11

[42] Schilling J, Müller F, Matthias S, Wehrspohn R B, Gösele U and Busch K 2001 Three-dimensional photonic crystals based on macroporous silicon with modulated pore diameter Appl. Phys. Lett. 78 1180-2

[43] Föll H, Christophersen M, Carstensen J and Hasse G 2002 Formation and application of porous silicon Mater. Sci. Eng. R 39 93-141

[44] Schilling J and Scherer A 2005 3D photonic crystals based on macroporous silicon: towards a large complete photonic bandgap Photonics Nanostruct. Fundam. Appl. 3 90-5

[45] Huisman S R, Nair R V, Woldering L A, Leistikow M D, Mosk A P and Vos W L 2011 Signature of a threedimensional band gap observed on silicon inverse woodpile photonic crystals Phys. Rev. B 83205313

[46] Geim A K and Novoselov K S 2007 The rise of graphene Nat. Mater. 6 183-91

[47] Huisman S R, Nair R V, Hartsuiker A, Woldering L A, Mosk A P and Vos W L 2012 Observation of subBragg diffraction of waves in crystals Phys. Rev. Lett. 108 083901
[48] Cassagne D, Jouanin C and Bertho D 1996 Hexagonal photonic-band-gap structures Phys. Rev. B 53 7134-42

[49] Sözüer S and Haus J W 1993 Photonic bands: simple-cubic lattice J. Opt. Soc. Am. B 2 296-302

[50] Woldering L A, Mosk A P and Vos W L 2014 Design of a 3D photonic band gap cavity in a diamond-like inverse woodpile photonic crystal Phys. Rev. B 90115140

[51] Anderson P W 1958 Absence of diffusion in certain random lattices Phys. Rev. 1091492

[52] Ledermann A, Renner M and von Freymann G 2015 Threedimensional photonic quasicrystals and deterministic aperiodic structures Light Localisation and Lasing: Random and Quasi-Random Photonic Structures ed M Ghulinyan and L Pavesi (Cambridge: Cambridge University Press) ch 7

[53] Suzuki K, Kitano K, Ishizaki K and Noda S 2014 Threedimensional photonic crystals created by single-step multidirectional plasma etching Opt. Express 22 17100-6

[54] John S 1987 Strong localization of photons in certain disordered dielectric superlattices Phys. Rev. Lett. 58 2486-9

[55] Chutinan A, John S and Toader O 2003 Diffractionless flow of light in all-optical microchips Phys. Rev. Lett. 90123901

[56] Ishizaki K, Koumura M, Suzuki K, Gondaira K and Noda S 2013 Realization of three-dimensional guiding of photons in photonic crystals Nat. Photonics 7 133-7 